\title{
Resumen
}

El presente artículo presenta el análisis de los cambios en la participación y retornos de los accionistas producto de la combinación de negocios, aplicando un caso de estudio D\&S, tras su combinación con Walmart, entre el año 2008-2011. Además, identificar los posibles cambios en los porcentajes de participación y retornos de los mayores accionistas del caso de estudio, comparar las utilidades distribuibles y los dividendos repartidos de los accionistas mayoritarios, categorizar a los accionistas mayoritarios, de acuerdo a sus porcentajes de participación, antes y después del proceso de adquisición, año 2008 - 2011 y, por último, contrastar la participación y los retornos de los accionistas, producto de la combinación de negocios a través del caso de estudio. Se finalizará concluyendo que una combinación de negocios es un medio que le permite a la empresa obtener beneficios competitivos, una nueva forma de reestructuración, teniendo cambios en los porcentajes de participación y en las decisiones de los accionistas.

Palabras Claves: Combinación de Negocios, Cambios en la Participación, D\&S , Walmart, Retornos Accionistas, Accionistas Mayoritarios.

\section{LA COMBINACIÓN DE NEGOCIOS Y LOS INVERSIONISTAS: CASO DE ESTUDIO D\&S / WALMART (2008-2011).}

\section{BUSINESS COMBINATION AND INVESTORS: CASE STUDY D\&S / WALMART (2008-2011).}

\author{
Maritza Puente Martinez ${ }^{1}$
}

\begin{abstract}
This article presents an analysis of the changes in participation and returns of the shareholders as a result of business combination. It was studied taking into account the case of D\&S after its union with Walmart between 2008-2011. It identifies the possible changes in the percentages of participation and returns of the major shareholders, compares the distributable profits and dividends divided among them, categorizes the main shareholders according to their participation before and after the acquisition process, and finally, contrasts their participation and returns as a result of business combination involved in this case. To conclude we can say that business combination is a means which allows an enterprise to obtain competitive benefits and a new form of restructuring leading to changes not only in the percentages of participation but also in the decisions that shareholders make.
\end{abstract}

Key Words: Business Combination, Changes in participation, D\&S/ Walmart, Shareholders returns, major shareholders. 


\section{INTRODUCCIÓN}

Dentro de un grupo económico se toman decisiones para modificar la naturaleza jurídica de las empresas que lo componen, esto con el objetivo de darle eficiencia a la actividad, hacer más rentables los proyectos, mantenerse en los mercados o tener un mejor retorno para los accionistas. Hoy, los inversionistas de otros países están buscando un crecimiento, por ello buscan colocar sus capitales en otros países, y muchos miran a Chile, producto de la inestabilidad que ha tenido la economía mundial. Sin embargo, Chile ha demostrado ser una base para expandirse a los demás mercados latinoamericanos, principalmente, debido a su estabilidad institucional.

No siempre una fusión puede resultar perfecta, 0 en caso contrario puede ser el resultado de una de las mejores decisiones que puedan haber realizado los inversionistas. Aún con lo anterior, estos se ven afectados en sus porcentajes de participación, de ganancias, puesto que ya no son en muchos casos mayoritarios o dueños de la empresa, son el nuevo grupo entrante los que toman el mayor control y, por ende, las riendas de la empresa.

Lo expresado orienta la investigación al análisis de la situación de los inversionistas, en cuanto a su participación y retornos, tras el ideal de realizar una combinación de negocios. La investigación es desarrollada a través de un caso de estudio el que corresponde a la unión entre D\&S y Walmart, entre los períodos 2009 a 2011, para revisar la situación de los inversionistas, durante el proceso de adquisición. 


\section{COMBINACIÓN DE NEGOCIOS}

La combinación de negocios es un método de inversión, sobre la cual una empresa espera obtener algún rendimiento a futuro. Existen diferentes tipos de inversión, algunas son temporales y otras permanentes, entre éstas últimas esta el caso de la combinación de negocios.

La normativa chilena tiene su tratamiento en el BT\#72, mientras que en el ámbito internacional, de convergencia, tiene su tratamiento en la NIIF3 y la NIC22, todas reflejan el tratamiento que debe tener la combinación de negocios.

Una combinación de negocios puede estructurarse de diversas formas:

- $\quad$ Mediante la compra de acciones de una empresa

- La compra de activos netos

- $\quad$ La emisión de nuevas accio-

nes

Por transferencia de efectivo, efectivo equivalente $u$ otros activos (BT72, 2004, 6)

Estas situaciones pueden transarse entre los socios 0 accionistas de las empresas participantes de la combinación de negocios 0 , también, entre los accionistas o socios de otra empresa.

El surgimiento de una combinación de negocios se dará cuando una entidad adquiera un parte 0 el total de otra entidad. También se puede entender como aquellas operaciones donde una empresa adquiere el control de uno 0 más negocios. La normativa también entrega definiciones sobre combinación de negocios.

Por combinación de negocios, se ha de entender:

-"es la unificación de empresas independientes en una entidad económica única, como resultado de que una de las empresas se une con la otra u obtiene el control sobre los activos netos y las operaciones de la misma." (NIC22, 1993, 4)

-"es cuando mediante una adquisición 0 una unificación de intereses las operaciones de una empresa se integran con las operaciones de otra u otras empresas, las que pueden desarrollar negocios similares, integrados vertical/ horizontalmente o totalmente distintos." (BTำ2, 26)

-“La unión de entidades o negocios separados en una única entidad que informa." (NIIF3, 23)

Otras definiciones necesarias para comprender el concepto de combinación de negocios son:

-Control: "es el poder para dirigir las políticas de explotación y financieras de una empresa, con el fin de obtener beneficios de sus actividades." (NIC22, 1993, 4)

-Matriz: "aquella entidad que tiene una 0 más afiliadas." (NIIF3, 24)

-Afiliada: "Una entidad incluyendo una sociedad de personas, que es controlada por otra conocida como matriz." (NIIF3, 24)

\section{FUSIÓN DE EMPRESAS.}

El crecimiento es un proceso esencial para el éxito y la vitalidad de las empresas, sin éste tendrían problemas en la búsqueda de los logros y la atracción de directores competentes.

La decisión que lleva a las entidades a realizar una fusión, es una decisión de inversión, que en muchos casos es estratégica, o también una manera de salvar la compañía. Sin embargo, cuando una empresa opta por esta alternativa es necesario conocer el tipo de fusión a la que se somete.

"La fusión consiste en la reunión de dos o más sociedades en una sola que las sucede en todos sus derechos y ob- 
ligaciones, y a la cual se incorporan la totalidad del patrimonio y accionistas de los entes fusionados". (Ley 18.046 Sociedades Anónimas, 1981, 99).

\section{TIPOS DE FUSIÓN .}

Según lo señalado por el Colegio de Contadores de Chile S.A (2002), en el boletín técnico $\mathrm{N}^{\circ} 72$, existen 3 tipos de fusiones:

Figura 1: Fusión de Sociedades

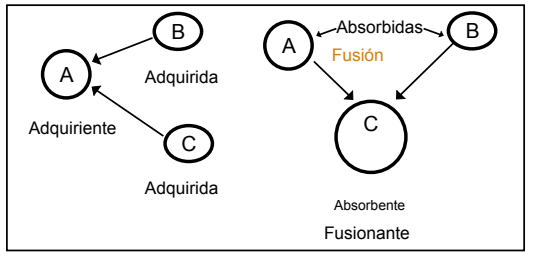

Fuente: Barrera, 2010, 117

a) Fusión por absorción: Una empresa adquiere la totalidad de las acciones 0 derechos de otra, la que resulta legalmente disuelta y absorbida por la compradora.

b) Fusión por incorporación: Una empresa recibe como aporte de capital la totalidad de los activos y pasivos de otra. La primera aumenta consecuentemente su capital y la entrega a los dueños de la segunda la correspondiente participación en el capital de la continuadora.

c) Fusión por creación: Menos común es el caso en que los activos y pasivos de dos o más empresas son transferidos a una nueva entidad, produciéndose la disolución de las de origen. (Colegio de Contadores de Chile A.G., 2002, 6)

\section{VALORES DE LAS EMPRESAS TRAS LA FUSIÓN.}

Llevar a cabo una fusión necesita de una adecuada planificación para conseguir los objetivos. Las empresas buscan revalorizar todas sus áreas de producción, esto como parte de la estrategia de fusión. Garrido (2004) presenta una serie de valores a considerar por las empresas producto de una fusión, estos son:

\section{VALOR ECONÓMICO}

- Competencia: Es común que producto del proceso de fusión exista un desconocimiento, por parte del mercado, de la nueva entidad, pudiendo darse una alteración en la atención de los clientes, además de que una parte del mercado se perderá producto de la combinación, favoreciendo a la competencia. (Garrido, 2004)

- Clientes: Tal como se señalará, al hablar de una situación desventajosa existe implicaciones culturales, que provocan que los clientes se vean afectados por esta transacción, ya que el mercado pierde oportunidades que antes le permitía a los clientes tener más lugares donde elegir, sin embargo, ahora ya no contarían con una completa variedad de productos, ni de lugares. (Garrido, 2004)

- Recurso Humano: Cada empresa le entrega un valor diferente al recurso humano, teniendo presente que son las personas las que hacen operativas a las empresas. Pero así como para algunos es un factor fundamental, para otros es un factor que se reemplaza por nuevas tecnologías, un bien no del todo necesario. (Garrido, 2004).

\section{VALOR CONTABLE.}

En la normativa chilena, el tratamiento contable de las fusiones se veía reflejado en el BT. 72 del Colegio de Contadores de Chile S.A., que trata sobre la "Combinación de Negocios". En la ac- 
tualidad el país ya se encuentra inmerso en un proceso de convergencia, donde las empresas se acomodan a la norma internacionales de contabilidad. En materia de fusión, el tratamiento contable de las entidades combinadas se encuetra en la NIC N²2 denominada Combinación de Negocios.

\section{VALOR TRIBUTARIO.}

- Costo tributario de activos y pasivos de la sociedad fusionada en la sociedad fusionante: El costo que debe ser considerado en la entidad fusionante, es el mismo costo de la sociedad fusionada al momento de la combinación. El SII, en esta materia señala, en la Circular $\mathrm{N}^{\circ}$ 68 (1996), la necesidad de mantener registrado el valor tributario que tenían los bienes de la sociedad dividida, para acreditar el cumplimiento de las normas.

- Valor de adquisición de las acciones de canje, recibidas en la sociedad fusionante: Las acciones de canje recibidas son la prolongación jurídica de las antiguas. Determinar su valor permitirá, luego, el cálculo del mayor valor afecto a tributación, si éstas se enajenan.

- Valor tributario de los bienes adquiridos a considerar en la sociedad incorporante: El costo que se le da a los bienes, conforme a lo establecido en la Ley de Impuesto a la Renta, en su art. 30 , estará dado por el precio de adquisición, según lo establecido en el contrato o la factura, además del valor de los fletes y seguros, si así se dispone.

\section{VALOR FINANCIERO.}

Existen diferentes valores para una empresa, así como también diferentes métodos para valorarla. Un adquirente deberá pagar, un precio superior al valor de mercado, por las acciones de la empresa adquirida.
- Valorización de acciones a través de Ios beneficios: El valor de la empresa se refleja por la capitalización que hace el mercado de los beneficios que ésta tiene. Dicha relación es la que se conoce como el ratio PER, relación precio / ganancia.

- Valorización de acciones a través de los flujos de caja: El valor de los activos financieros es igual a la suma de los valores de caja que se pretende generar. Por ello, es que los expertos señalan que el método para obtener una aproximación de un valor, es el método de flujo de caja. (Garrido, 2004).

\section{TOMA DE CONTROL DE UNA SOCIE- DAD.}

Un mecanismo de control muy utilizado es la realización de una Oferta Pública de Acciones (OPA) es que la empresa que adquiere puede hacer una oferta a la empresa que desea adquirir.

La oferta se puede entender como una "propuesta de adquisiciones de acciones a determinado precio, muchas veces para lograr el control de la compañía. Con frecuencia, la oferta la hace otra compañía, casi siempre por encima del precio de mercado" (Van, Wachowicz, 2002, 640).

Esta medida sirve en ocasiones como una medida de precisión, que permite evitar un trato directo con los directores. Ahora bien, este método sirve también en los casos en que no hay negociaciones, donde simplemente se desea adquirir otra entidad. La oferta es pública en los medios de comunicación escrita, para dejar constancia de las intenciones de la empresa adquirente.

Para adquirir la compañía que se desea, se puede recurrir a diferentes tácticas. Una de ellas es la oferta, aunque también puede encontrarse la oferta de dos propuestas, que consiste en sugerir un precio superior al de la primera propu- 
esta.

La mayoría de los países coinciden en que las ofertas deben ser:

$\checkmark \quad$ Ofertas públicas
$\checkmark \quad$ Acciones que se
encuentren en el mercado
bursátil
$\checkmark \quad$ Deben tener un
precio superior al cotizado
$\checkmark \quad$ Válidas por un
período de tiempo establecido.

Pero las OPAS no son la única forma de tomar el control. En el caso de que un adquirente deseará tomar el control de la entidad adquirida, simplemente podría comprar títulos de acción de la empresa que desea adquirir, hasta alcanzar el porcentaje de acciones que le permita controlar las políticas y procedimientos del nuevo ente.

\section{LA COMBINACIÓN DE NEGOCIOS EN CHILE.}

Las empresas para desarrollarse en esta economía cambiante, que evoluciona constantemente, se colocan en la interrogante de decidir si vincularse a una estrategia competitiva o cerrar sus puertas, es lo que se denomina combinación de negocios.

En Chile, la combinación de negocios ha sido regulada a través de la Ley de Sociedades Anónimas y, de manera contable, en varias resoluciones técnicas, boletines y normativas.

Por lo general, los accionistas desean maximizar el valor de sus acciones y en cuanto estos deciden optar por la fusión, las ganancias que se producen tras el solo anuncio de la operación, elevan el valor de las acciones. Sin embargo, si este proceso no se llegara a concretar, el valor de las acciones volvería hasta los límites alcanzados, antes de dicho anuncio.
Por lo tanto, lo que buscan hoy los inversionistas es incursionar en nuevos mercados, que sirvan de salvavidas en tiempo de crisis y, es por ello, que hoy el número de transacciones no va en descenso, si no que va en aumento, con niveles más altos cada día.

\section{CASO DE ESTUDIO D\&S Y WALMART (2008-2011) D\&S .}

Es una compañía chilena, domiciliada en la ciudad de Santiago de Chile. Sociedad anónima abierta, constituida, según escritura pública el 17 de septiembre del año 1985, dedicada a la distribución de alimentos y a la entrega de servicios. Con una experiencia de más de 50 años, mostrando un liderazgo basado en las estrategias de mejoramiento de su plan de negocios.

Su estrategia es mantener el liderazgo, a través del crecimiento, puesto que es la empresa líder en distribución de alimentos en el país, pero, además, busca un crecimiento en otros mercados y obtener mayores rentabilidades para sus accionistas.

El capital accionario de D\&S es de 6.520 millones de acciones distribuida en 1.213 accionistas, esto al 31 de Diciembre del 2008. Las acciones son transadas en la Bolsa de Comercio de Santiago, al 31 de Diciembre 2008. Los controladores de la compañía son la Familia Ibáñez, quienes poseían un $63,43 \%$ del total accionario.

En cuanto a competitividad, D\&S, posee tres áreas de negocios: supermercados, inmobiliarias y servicios financieros, la más importante en cuanto a las ventas, es el retail. Su estrategia se basa en la mejor relación entre precios y calidad, además de promociones con la tarjeta Presto, el aumento de su participación por segmentos, ofertas y el hacer mayor uso de las marcas propias. 


\section{WALMART.}

Compañía de origen norteamericano, con sede en el estado de Bentonville (Arkansas), fundada en 1962 por Sam Walton. Más del 40\% de los derechos de capital de la empresa se encuentran en manos de la familia Walton, a excepción de éstos ninguna persona tiene más de un $5 \%$ del total de acciones emitidas. Dedicada a la explotación de tiendas y almacenes de ventas al detalle alrededor del mundo.

\section{INVERSIONES AUSTRALES TRES LIMITADA (Inversiones Australes).}

Inversiones Australes es una sociedad de responsabilidad limitada. Constituida el 20 de octubre de 2008, según escritura pública, en Santiago de Chile. El objeto de esta sociedad es "efectuar inversiones en bienes muebles, corporales 0 incorporales, acciones de sociedades, derechos en otras sociedades, bonos, efectos de comercio y demás valores mobiliarios, todo ello tanto en Chile como en el extranjero..." (Prospecto de oferta pública IM trust "pdf", 2008, 5)

Inversiones Australes no participa de la propiedad de otras sociedades, pero tiene partes relacionadas, como lo son:

- Inversiones Australes Uno Limitada

- Inversiones Australes Dos Limitada

- WM SARHCO III, LLC

- WM Latin American Holdings (BVI) III Corp.

- WM Latin American Holdings (BVI) II Corp.

Esta sociedad, Inversiones Australes, es filial de Walmart Stores, Inc. Walmart no participa ya sea de forma directa 0 indirecta de propiedades que son fiscalizadas por la SVS, lo que hace es invertir en fondos de administración por terceros, los cuales pueden tener inversiones en sociedades fiscalizadas por la SVS, en estos casos ni sus sociedades relacionadas tienen influencia alguna en las decisiones de inversión de tales fondos, así como los derechos a votos a dichas inversiones.

\section{TRANSFORMACIÓN DE D\&S A WALMART CHILE S.A.}

D\&S, fue fundada por la familia Ibáñez, una familia de políticos, académicos y empresarios chilenos que tenían el control de la compañía. Desde hace mucho tiempo tenían planes para la compañía, planes de internacionalización, entre ellos estuvo una fallida fusión con Falabella el año 2008, fue el tribunal de la libre competencia que trancó sus aspiraciones. (Memoria D\&S, 2008,105)

Sin embargo, sus planes de internacionalización no se vieron truncados por completo puesto que el 19 de diciembre de 2008, cerraron negociaciones con la empresa multinacional estadounidense Walmart (compañía de los Walton), el mayor minorista del mundo. De esta manera, el 23 de diciembre de 2008, Walmart, a través de su filial Australes Tres Limitada (Inversiones Australes), publicó en la prensa chilena un aviso de su intención de tomar el control de D\&S. Esto, mediante el lanzamiento de una oferta pública de adquisición de acciones.

La oferta que lanzó fue de US\$ 0,408 por acción, operación que valorizó a la empresa en US\$ 2.660 millones, la OPA se lanzó para obtener el $100 \%$ del holding de D\&S.

La Familia Ibáñez controla el 63,4\% de la compañía, hasta antes de la adquisición de acciones por parte de Walmart. Así es como el 29 de Enero de 2009, tras esta exitosa OPA, Walmart toma el control de D\&S. 
Al 31 de enero de 2009, el control de Walmart alcanza un $52,57 \%$ de las acciones de la firma, quedando el restante de acciones en manos de los Ibáñez y accionistas minoritarios.

El 23 febrero de 2009, su filial Inversiones Austral, lanza una segunda OPA, dando aviso al mercado y al público en general de sus intenciones. A marzo del mismo año Walmart había adquirido como tenedor directo e indirecto 4.860.718.212 acciones, quedando como propietario de D\&S con un porcentaje de participación en la firma del $74,55 \%$ del total de acciones de la compañía, un $25,07 \%$ de las acciones se mantiene en manos de los Ibáñez, mientras que un $0,38 \%$ en manos de accionistas minoritarios.

En mayo del 2010, Walmart tiene el control del $74,61 \%$ de D\&S. Este mismo año se llevaría a cabo un gran cambio de la compañía. Durante el mes de noviembre de 2010, se acordó por unanimidad el someter a una junta extraordinaria de accionistas el cambio de la razón social de la compañía, es por ello que, tras una junta extraordinaria de accionistas realizada el día 22 de noviembre de 2010, dicho cambio fue aprobado poniéndolo en marcha, de esta manera la compañía paso de Distribución y Servicios S.A (D\&S), a Walmart Chile S.A.

Para el año 2011, los cambios en la compañía continuaron, la división de servicios financieros cambia su razón social a Walmart Chile, Servicios Financieros $S . A$, mientras que la división inmobiliaria cambia a Walmart Chile Inmobiliaria S.A. Por su parte, los Hiper Lider cambian su logo y nombre por Lider. (Memoria Walmart Chile, 13, 2011)

Un total de 325 accionistas son los que posee Walmart Chile al 31 de diciembre del 2011, siendo Walmart el propietario a dicha fecha del $74,65 \%$ del to- tal de sus derechos de capital, puesto que es tenedor directo e indirecto de 4.867.453.738 de acciones emitidas.

\section{METODOLOGÍA.}

El presente artículo se basa en la investigación realizada bajo una metodología cuantitativa con un alcance descriptivo.

Se determina la empresa en estudio por método de muestreo por conveniencia, según los siguientes criterios:

1- Combinación de
Negocios
$2-\quad$ Combinación de
negocios en Chile
3- Antecedentes caso
de estudio

\section{Variables del estudio}

\section{Variable 1}

"Participación de los accionistas"

\section{Definición conceptual:}

Porcentaje que refleja el grado de control que posee el accionista sobre la entidad, y que le da derecho a obtener dividendos.

\section{Definición operacional:}

Revisión y análisis, a través de cuadros comparativos, que permitan conocer la participación actual y anterior al proceso de combinación de negocios.

\section{Variable 2}

"Retornos obtenidos por los accionistas, antes y después del proceso de adquisición, aplicados al caso de estudio"

\section{Definición conceptual}

Son las ganancias obtenidas por los accionistas, tras los resultados de la compañía, al cierre de las operaciones y por sus porcentajes de participación. 
Definición operacional

Se conocerá la situación de los retornos de los accionistas a través de cuadros comparativos.

\section{RESULTADOS.}

El estudio contiene un período de evaluación para el análisis de los resultados desde el año 2008 a 2011 períodos que se tomaron para el análisis del caso de estudio, la combinación de negocios entre D\&S y Walmart.

Durante el proceso de revisión, se pudo observar el cambio en la participación del grupo que hasta finales del 2008 poseía el control de la compañía D\&S. El grupo Ibáñez controlaba la compañía con un $63,48 \%$ del total acciones de la empresa, quienes conformaban este porcentaje de acciones son:

$$
\begin{aligned}
& \text { - Scott } \\
& \text { - Señor Felipe lbáñez } \\
& \text { Atkinson Young (cónyuge de } \\
& \text { Felipe Ibáñez) } \\
& \text { - Señora Heather } \\
& \text { Ibáñez Scott }
\end{aligned}
$$

De la memoria de la compañía del año 2008 se pudo obtener la información, sobre qué empresas controlaba este grupo, y con las cuales tenía el control directa e indirectamente en D\&S.

Felipe Ibáñez cuenta con una participación de un $17,22 \%$ del total accionario, que equivalen a 1.122.730.737 acciones, divididas en 3 compañías, que le permiten tener una parte del control de la empresa. Heather Atkinson, por su parte, refleja un porcentaje de $14,01 \%$, equivalente a 913.601.867 acciones de la compañía, de tan solo una empresa con la cual tiene una relación directa e indirectamente en el control. Don Nicolás Ibáñez que tiene un $31,96 \%$, equivalente a 2.084.115.792 acciones, dividido en ocho empresas con las cuales tiene esta participación en el capital accionario de forma directa e indirecta. Y, por último, la participación conjunta, a través de una empresa de Felipe y Nicolás Ibáñez, con la cual tienen un porcentaje de $0,29 \%$ equivalente a 19.007 .676 acciones. En total los Ibáñez controlan 4.139.456.072 acciones que equivalen a un $63,48 \%$ del capital accionario, que les permite ser el grupo controlador de la compañía hasta ese entonces (2008).

Esta situación duró hasta enero del año 2009, es desde ese momento cuando la OPA lanzada por Inversiones Australes se hizo efectiva y adquirió un poco más del $50 \%$ de las acciones de D\&S, convirtiéndose en los nuevos controladores. 


\section{Cuadro №1: Participación del grupo Ibáñez desde}

el año 2008 a 2011.

\begin{tabular}{|c|c|c|c|c|c|c|c|}
\hline & Accionistas & $\begin{array}{c}2008 \\
\text { diciembre }\end{array}$ & $\begin{array}{l}2009 \\
\text { enero }\end{array}$ & $\begin{array}{r}2009 \\
\text { abril }\end{array}$ & $\begin{array}{c}2009 \\
\text { diciembre }\end{array}$ & $\begin{array}{c}2010 \\
\text { diciembre }\end{array}$ & $\begin{array}{r}2011 \\
\text { diciemb } \\
\end{array}$ \\
\hline Grupo Ibáñez & $\begin{array}{l}\text { Rentas HAY y Compañia, } \\
\text { Sociedad Colectiva Civil, }\end{array}$ & 14,01 & & & & & \\
\hline & Retail International S.A & 15,2 & 15,2 & & & & \\
\hline & Retail International tres S.A. & 3,39 & 3,39 & & & & \\
\hline & $\begin{array}{l}\text { Inversiones International Supermarket } \\
\text { Holdings Limitada }\end{array}$ & 3,12 & & & & & \\
\hline & $\begin{array}{l}\text { Rentas Fis y Compañía Sociedad } \\
\text { Colectiva Civil }\end{array}$ & 14,01 & 14,01 & 10,53 & 10,53 & 10,53 & 10,53 \\
\hline & $\begin{array}{l}\text { Servicios Profesionales y de } \\
\text { Comercialización Cuatro Ltda. }\end{array}$ & 1,65 & 1,65 & 1,65 & 1,65 & 1,65 & 1,65 \\
\hline & $\begin{array}{l}\text { Servicios Profesionales y de } \\
\text { Comercialización Dos Ltda. }\end{array}$ & 1,65 & 1,65 & 1,65 & 1,65 & 1,65 & 1,65 \\
\hline & Schouton N V Agencia en Chile & 9,54 & 0,15 & 0,15 & 0,15 & 0,15 & 0,15 \\
\hline & \begin{tabular}{|l|} 
Rentas Hay y Compañia, Sociedad \\
Colectiva Civil \\
\end{tabular} & & 4,02 & & & & \\
\hline & Shouten Inversiones Civiles Limitada & & & 11,09 & & & \\
\hline & Total & 62,57 & 40,07 & 25,07 & 13,98 & 13,98 & 13 \\
\hline
\end{tabular}

Fuente: Elaboracion Propia, 2012.

Desde el lanzamiento de la primera OPA, D\&S tiene nuevos controladores "Walmart" quien posee el control indirectamente de una serie de sociedades que se conglomeran en Inversiones Australes. Cuadro N02.

Cuadro №2: Sociedades pertenecientes a Inversiones Australes.

\begin{tabular}{|c|c|c|c|c|c|}
\hline $\begin{array}{l}\text { Socios de Inversiones } \\
\text { Australes }\end{array}$ & $\%$ participación * & Socios & $\%$ part. & Socios & $\%$ pa \\
\hline $\begin{array}{l}\text { 1.- Inversiones Australes } \\
\text { Uno Limitada }\end{array}$ & \multirow[t]{3}{*}{$99,9 \%$} & $\begin{array}{l}1.1 \text { Inversiones Australes } \\
\text { Dos Limitada }\end{array}$ & $99,9 \%$ & \multirow{2}{*}{$\begin{array}{l}\text { 1.1.1 WM Latin American } \\
\text { Holdings (BVI) II Corp. } \\
\text { 1.1.2 WM Latin American } \\
\text { Holdings (BVI) II Corp. } \\
\end{array}$} & \multirow{2}{*}{$\begin{array}{r}99, \\
0,\end{array}$} \\
\hline & & & & & \\
\hline & & 1.2 WM SARHCO III, LLC & $0,1 \%$ & & $100^{\prime}$ \\
\hline 2.- WM SARHCO III, LLC & $0,1 \%$ & & $100 \%$ & & \\
\hline
\end{tabular}

Fuente: Elaboración Propia, 2012. 
Inversiones Australes filial de Walmart Store Inc tiene el control de D\&S actualmente Walmart Chile S.A., de forma directa e indirecta, a través

de Inversiones Australes Uno Limitada y WM SARHCO III, LLC., como se puede observar en el cuadro №3.

Cuadro №3: Comparativo de los 12 mayores accionistas al 31 de Diciembre de 2008 a 2011.

\begin{tabular}{|c|c|c|c|c|c|}
\hline \multirow[t]{2}{*}{ Propietarios } & & 2008 & 2009 & 2010 & 201 \\
\hline & Accionistas & $\%$ & $\%$ & $\%$ & $\%$ \\
\hline \multirow[t]{2}{*}{ Inversiones Australes } & Inversiones Australes Tres Limitada & 0 & 74,61 & 74,61 & 74,6 \\
\hline & Total Inversiones Australes & 0 & 74,61 & 74,61 & 74,6 \\
\hline \multirow[t]{9}{*}{ Grupo lbáñez } & Rentas HAY y Compañía, Sociedad Colectiva Civil & 14,01 & & & \\
\hline & Retail International S.A & 15,2 & & & \\
\hline & Retail International tres S.A. & 3,39 & & & \\
\hline & Inversiones International Supermarket Holdings Limitada & 3,12 & & & \\
\hline & Rentas Fis y Compañia Sociedad Colectiva Civil & 14,01 & 10,53 & 10,53 & 10,5 \\
\hline & Servicios Profesionales y de Comercialización Cuatro Ltda. & 1,65 & 1,65 & 1,65 & $1,6 !$ \\
\hline & Servicios Profesionales y de Comercialización Dos Ltda. & 1,65 & 1,65 & 1,65 & $1,6 !$ \\
\hline & Schouton N V Agencia en Chile & 9,54 & 0,15 & 0,15 & $0,1 !$ \\
\hline & Tatal Cruinn lháñns & a) 57 & 1208 & 1200 & 120 \\
\hline
\end{tabular}

\begin{tabular}{|c|c|c|c|c|c|}
\hline \multirow[t]{3}{*}{ Otros. } & $\begin{array}{l}\text { J.P. Morgan Chase Bank (Depositario de ADRs) } \\
\text { Fondo de Inversiones Privado la Querencia } \\
\text { AFP Provida S.A. (Fondo C) } \\
\text { Banchile Corredora de Bolsa S.A. } \\
\text { Fondo Inversión Privado Aurora III } \\
\text { Larraín Vial S.A. Corredora de Bolsa } \\
\text { Santander S.A. Corredora de Bolsa S.A. } \\
\text { Findel Westermeier Alicia } \\
\text { Consorcio Corredora de Bolsa S.A. } \\
\text { Corp. Capital Corredora de Bolsa S.A. } \\
\text { Chile Market S.A. Corredora de Bolsa } \\
\text { Corpbanca Corredora de Bolsa S,A, } \\
\text { M Trust S.A. Corredores de Bolsa } \\
\end{array}$ & $\begin{array}{l}4,12 \\
2,06 \\
1,74 \\
2,52\end{array}$ & $\begin{array}{c}0,08 \\
11,09 \\
0,06 \\
0,03 \\
0,01 \\
0,01 \\
0,01\end{array}$ & $\begin{array}{c}0,07 \\
11,09 \\
0,09 \\
0,03 \\
0,01 \\
\\
0,01 \\
0,01\end{array}$ & $\begin{array}{r}0,0 \\
11,0 \\
0,0 ! \\
0,0 \\
0,0 \\
0,0 \\
0,0 \\
\end{array}$ \\
\hline & Total Otros & 10,44 & 11,29 & 11,31 & 11,3 \\
\hline & Otros, minoritarios & 26,99 & 0,12 & 0,1 & 0 \\
\hline
\end{tabular}

Fuente: Elaboración Propia, 2012. 
El capital accionario de Walmart Chile S.A., durante todo el período de estudio, se encontró en constante cambio de participaciones, entre las diferentes OPAs y traspasos de acciones que se fueron realizando. De los datos de las memorias se tomaron los cuadros de participación de los 12 mayores accionistas de la compañía, así como información de porcentajes de participación y del grupo accionario total que posee la empresa.

Las utilidades distribuibles obtenidas por la compañía, durante el período de estudio, fueron las siguientes:

- año 2008: 20.998.308

- año 2009: -48.937.545

- $\quad$ año 2010: 61.373.398

- año 2011: 113.904.520

Esto en cuanto a utilidades, mientras que los dividendos distribuidos fueron:

\begin{tabular}{|r|r|r|}
\hline Año & M\$ & \multicolumn{1}{|c|}{ \$/Acción } \\
\hline 2008 & 38.772 .426 & 6 \\
2009 & 13.040 .000 & 2 \\
2010 & 32.600 .000 & 5 \\
2011 & 32.955 .000 & 5.054 \\
\hline
\end{tabular}

Fuente: Elaboración Propia, 2012.

El cuadro con la información de los dividendos distribuidos, como de las utilidades distribuibles, fueron obtenidos también a través de las memorias de la compañía en estudio.

\section{DISCUSIÓN DE RESULTADOS}

Los resultados obtenidos a través del análisis del caso de estudio se pudieron obtener gracias a las memorias de la compañía y la información recopilada a través de diversos medios escritos, internet y libros, entre otros.
El primer paso fue ver que el caso de estudio es o no una combinación de negocios. El marco teórico, al respecto, señala que una combinación de negocios puede generarse por medio de una fusión o la adquisición de elementos patrimoniales de una empresa y que, entre las formas de estructuración de una combinación de negocios, se encuentra la compra de acciones de una empresa y la emisión de nuevas acciones. D\&S, fue adquirida por Walmart mediante una adquisición de acciones de la compañía, esto por medio del lanzamiento de OPAs, por ello, este caso de estudio se considera una combinación de negocios.

Una combinación de negocios se puede entender, además, como una operación donde una empresa adquiere el control de uno 0 más negocios y el control se tiene cuando la compañía que adquiere posee más del $50 \%$ de las acciones de la compañía adquirida. En este caso de estudio, a la fecha, Walmart posee un $74,65 \%$ de las acciones de D\&S, tomando el control de la compañía con más del $50 \%$ de las acciones, quedando el resto del porcentaje accionario en manos de los antiguos controladores de la compañía: el grupo Ibáñez y los accionistas minoritarios.

Esta combinación de negocios no solo trajo cambios de tipo accionario, en cuanto a participación de los accionistas, sino también en las utilidades que generaron estas acciones. Fue el año 2011 cuando se reflejó el mayor cambio, la utilidad fue de 17,47 pesos por acción, mientras que la menor utilidad se obtuvo el año 2009 ya comenzado el proceso de combinación de negocios con menos 7,50 pesos por acción. En cuanto a los precios de cierre de las acciones fue el año 2009 donde se vio el menor precio por 206 por acción, mientras que el año 2010 tras el mayor cambio de la compañía tras pasar de ser D\&S 
a llamarse Walmart Chile S.A., tuvo un precio de cierre de la acción de 328 pesos por acción, el mayor valor obtenido durante el período en estudio.

Las utilidades distribuibles de la compañía también reflejaron cambios notorios, al comienzo del cambio se generó un pérdida de $\mathrm{M} \$ 48.937 .545$, pero en los años siguientes, esta situación cambió totalmente, generando ya el año 2010 utilidades de $M \$ 61.373 .398$ y en el año 2011, esta situación logró casi al doble de la utilidad obtenida el año 2010, en M\$113.904.520.

Por su parte los dividendos distribuibles tuvieron una caída tan sólo en 2009, fue el período en que menos dividendos se distribuyeron, sin embargo, fue en 2008 el período donde más dividendos se distribuyeron con $\mathrm{M} \$ 38.772 .426$, los años 2010 y 2011 se mantuvieron casi con las mismas cantidades distribuidas.

En definitiva, los cambios más grandes del caso de estudio fueron los cambios accionarios en los porcentajes de participación, que trajo a un nuevo controlador de la compañía y los cambios en las utilidades, que comenzaron a generarse luego de un plan estructurado por los accionistas, para mejorar la compañía. Entre ellos el llevar a cabo el cambio de nombre de la empresa, actualmente Walmart Chile S.A. 


\section{CONCLUSIONES}

Competir con nuevos mercados, mejorar su participación en estos, o sobresalir por sobre sus demás competidores, son parte del nuevo ambiente económico cambiante. Continuamente, las organizaciones buscan nuevos métodos de restructuración, que le permiten a las empresas competir en el mercado local e internacional.

Para la realización del marco teórico se tomó información a través de libros, normativas, internet, tesis, entre otros. Mientras que para la elaboración del caso de estudio se tomó información de las memorias de la compañía, de sus sitios de internet, así como información contenida en la SVS y las publicaciones en los medios de prensa.

El objetivo de este estudio era comprobar si existirían cambios en la participación y retornos de los accionistas. Es por ello que se llevó a cabo el estudio de la combinación de negocios D\&S / Walmart, y se pudo concluir que:

* La adquisición, por parte de Walmart, sobre D\&S, resultó ser una combinación de negocios. Donde hubo un cambio accionario, por medio de diferentes OPAs, realizadas por Walmart para adquirir las acciones de D\&S y poder tomar el control de la compañía.

* Desde el comienzo de la combinación de negocios, se pudo observar el ingreso a la compañía de un nuevo controlador Walmart, con más del $50 \%$ del capital accionario de la compañía.

* Se pudo observar, además una disminución en la cantidad de miembros accionarios, de 1.213 accionistas en 2008 a 324 accionistas a marzo de 2012.

* En los porcentajes de participación de los accionistas, es donde se vieron los mayores cambios y esto se pudo observar, a través de los cuadros de participación de los 12 mayores accionistas, cuadros que se presentan en las memorias de la compañía.

* En cuanto a las utilidades, se generaron mayores utilidades distribuibles, pero no cambiaron mucho los porcentajes de distribución de dividendos. Sin embargo, al haber diferencias en cuanto a las participaciones de los accionistas, sí se puede observar que los retornos de los accionistas de la compañía adquirida se vieron disminuidos. La compañía, sin embargo, mantiene una participación en el mercado nacional alta, y con una presencia en los mercados internacionales, gracias a esta combinación de negocios, con la mayor minorista del mundo.

En definitiva, una combinación de negocios es un medio que le permite a la empresa obtener beneficios competitivos, una nueva forma de reestructuración, pero que a los accionistas de la compañía que ha sido adquirida le traerá cambios en los porcentajes de participación obtenidos, habrá nuevos accionistas, habrá otros accionistas que decidirán irse de la compañía, otros que cederán sus acciones, otros que se quedarán, pero que ya no tendrán la misma participación, ni los mismos retornos que tenían antes de la combinación de negocios. 


\section{BIBLIOGRAFÍA}

- $\quad$ Administrador (2011, 30 Marzo) La fusión de TAM y Lan. http://www. managementjournal.net/index.php?option= comt\&view =article\&id = 207: la-fusion-detam-y-lan\&catid=36:fusiones-adquisicione s\&ltemid =84\#comment-28

- Álvarez, J. (2006) Análisis crítico de la regulación de fusión y toma de control de empresas de Chile, cuando en el mercado existe alta concentración. Tesis para optar al grado de Contador Público Auditor. Escuela de Auditoría. Universidad de Valparaíso - Valparaíso. Chile.

- $\quad$ Araya, M. - Roco, F. (2005) La fusión de empresas y poder monopólico. Tesis para optar al grado de Licenciado en Ciencias Jurídicas. Escuela de Derecho. Universidad de Valparaíso - Valparaíso. Chile.

- $\quad$ Barrer, M. (2006) Combinación de negocios: una visión aplicada del boletín técnico No 72 en empresas de la $V$ región. Tesis para optar al grado de Contador Público Auditor. Escuela de Auditoría. Universidad de Valparaíso - Valparaíso. Chile.

- $\quad$ Barrera, P. (2010) Consolidación de Estados Financieros (PDF). Página 117.

http://es.scribd.com/doc/55768352/25/ Grafico-14-Adquisicion-de-sociedades

- Barril, R. (2002) Estudio de un caso de fusión de empresas y una alternativa estratégica para procesos administrativos. Tesis para optar al grado de Magister en Gestión de Organizaciones. Escuela de Graduados. Universidad de Valparaíso - Valparaíso. Chile.

- $\quad$ Becerril, J. (2004, Febrero) Fusión de sociedades: análisis de la fusión de AOL Time Warner. http://www.gestiopolis. com/recursos2/documentos/fulldocs/fin/ fusaoltime.htm

- $\quad$ Bertaggia, C. (2010) ABC-Din prevé elevar ventas $30 \%$ en 2010 sin cambiar su foco de negocios.

http://diario.latercera.com/2010/08/28/01/ contenido/negocios/10-36883-9-abcdinpreve-elevar-ventas-30-en-2010-sincambiar-su-foco-de-negocios.shtml

- $\quad$ Borredá, R. (1999) Fusiones y adquisiciones de empresas. Tesis para optar al grado de Ingeniero Comercial mención Administración. Escuela de Ingeniería Comercial. Universidad de Valparaíso Valparaíso. Chile.

- $\quad$ Cerda, F. (2009, 11 Marzo). Fusión Scotiabank y el Banco del Desarrollo: ejemplo de una mala gestión del «espíritu».

http://www.franciscocerda.cl/content/ view/463148/Fusion-Scotiabank-y-elBanco-del-Desarrollo-ejemplo-de-unamala-gestion-del-espiritu.html

\section{(}

Colegio de Contadores de Chile S.A (2002) Boletín técnico $n^{\circ} 72$.

Página 17

- Comité retail financiero (2010) Senado Rechaza Posible Fusión de Soprole con Nestlé. http://www. comiteretailfinanciero.cl/crf/noticias-deldia/senado-rechaza-posible-fusion-desoprole-con-nestle/

- Definanzas (2008, Febrero) Falabella y D\&S no podrán fusionarse todavía, Chile. http://definanzas. com/2008/02/02/falabella-y-ds-no-podranfusionarse-todavia-chile/

- $\quad$ Dmelgar (2011, 17 Abril) Combinación de Negocios. Archivado en http://www.buenastareas.com/ensayos/ Combinaciones-De-Negocios/305767.html

- Economía y Negocios (2007, Mayo) Fusión de Falabella y D\&S crea la mayor empresa del retail en América del Sur. http://www.economiaynegocios.cl/ noticias/noticias.asp?id $=26700$

- $\quad$ Emol (2010, Diciembre) Dueños de LAN Chile reorganizan sociedades para fusión con TAM. http://www.emol.com/ noticias/economia/detalle/detallenoticias. $\underline{\text { asp?idnoticia }=454368}$

- $\quad$ Empresa D\&S (2007 - 2010) Memoria anual. http://www.dys.cl/wps/ wcm/connect/dys/Dys/inversionistas/ informacion financiera/memorias estadosfinancieros/

- $\quad$ Estrategia (2011) Fusiones y Adquisiciones Podrían Crecer un 25\% en 2011. http://www.estrategia.cl/detalle 
noticia.php?cod $=37743$

- Garrido, D. (2004) La fusión de sociedades anónimas como instrumento para la revalorización tributaria de la empresa. Tesis para optar al grado de Contador Público Auditor. Escuela de Auditoría. Universidad de Valparaíso Valparaíso. Chile

- Instituto Valenciano de la Exportación "IVEX" (2011) Informe Chile 2011 (PDF). IVEX Chile, Santiago Chile.

www.ivex.es/dms/...pais/Chile.../ CHILEINFORME\%20PAIS\%202011.pdf

- Ley 18046 sociedades anónimas, Biblioteca del Congreso Nacional de Chile (1981) Artículos 57, 67, 69, 99, 100.

- Norma Internacional de Contabilidad № 22 (1983) Combinación de Negocios.

Página $4-5$.

- $\quad$ Norma de Información Financiera NIIF 3 (2008) Combinación de Negocios.

Página $4-7,25$

- Pinto, G. (2005) Fusión de empresas. Santiago: Lexis Nexis

Página 12, 26 - 27.

- Real Academia Española (2005) Diccionario real lengua española. http:/l buscon.rae.es/drael/

- Servicio de Impuestos Internos (1995) Circular 17 http://www.sii.cl/ documentos/circulares/1995/17.htm

- $\quad$ Silva, M. (2007) Unificación y síntesis en la aplicación de la legislación de la ley de la renta y la ley de impuestos al valor agregado en torno a las fusiones de sociedades. Tesis para optar al grado de Contador Público Auditor. Escuela de Auditoría. Universidad de Valparaíso Valparaíso. Chile.

- Van Home, J. - Wachowicz, J. (2002) Fundamentos de administración financiera. México: Pearson Educación. Capitulo 23, páginas $623-649$. 